\title{
On Differentially Demodulated CPFSK
}

\author{
Anthony Griffin and Desmond P. Taylor \\ griffiab@elec.canterbury.ac.nz and taylor@elec.canterbury.ac.nz \\ Electrical and Electronic Engineering \\ University of Canterbury \\ New Zealand
}

\begin{abstract}
This paper develops a differential encoder for differentially demodulated continuous phase frequency shift keying (CPFSK). CPFSK schemes with modulation index $h=\boldsymbol{F} / P$, where $\boldsymbol{F}$ and $P$ are relatively prime positive integers, can be represented by a decomposed model consisting of a continuous phase encoder (CPE) and a memoryless modulator (MM). The differential encoder is shown to fit well with the CPE and form a decomposed model of differentially encoded CPFSK (DCPFSK). A basic receiver structure for differentially demodulating DCPFSK is presented along with simulation results. An exact formula for the minimum squared Euclidean distance (MSED) of differentially demodulated DCPFSK is also given.
\end{abstract}

\section{Introduction}

Continuous Phase Modulation (CPM) is an attractive communication scheme as it is a true constant envelope modulation, and can be amplifed by amplifiers working in their non-linear regions. Continuous phase frequency shift keying (CPFSK) is a simple CPM scheme that is very useful due to its narrowband RF requirements. CPFSK can be decomposed into a two part model [1] that isolates the coding and modulation inherent within CPFSK. This model has allowed codes to be designed specifically for CPFSK with improved performance compared to previous schemes [2] [3].

CPM schemes are sensitive to phase jitter, and therefore require an accurate phase reference for coherent demodulation. Recovering the carrier accurately enough can be difficult in more extreme channels. A solution that avoids the need for a phase reference is differential demodulation, where the previous symbol is used to demodulate the current one. A differential CPFSK (DCPFSK) scheme has been developed by Yuan and Taylor [4], but their differential encoder was designed for bipolar binary CPFSK, and does not lend itself to coding. An $M$-ary differential encoder that interfaces well with the decomposition model would allow the development of a decomposed differential model, and codes to be designed especially for DCPFSK.

In Section 2 we describe CPFSK and its decomposition model with a modulation index of $h=\boldsymbol{F} / P$. In Section 3 we develop a differential encoder for CPFSK and present the decomposition of DCPFSK. We discuss coding DCPFSK, the differential phase trellis, and the squared Euclidean distance and spectrum of DCPFSK in Section 4. The results of some simulations of DCPFSK on the additive white Gaussian noise (AWGN) channel are presented in Section 5, and finally in Section 6 we draw some conclusions.

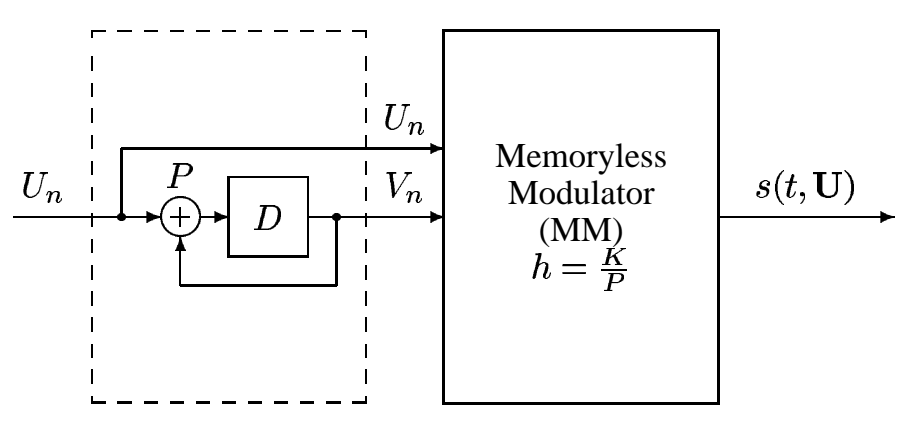

Continuous Phase Encoder (CPE)

Figure 1: Decomposition of CPFSK

\section{The Decomposition of CPFSK}

A CPFSK signal can be described [1] by

$$
s(t, \mathbf{U})=\sqrt{\frac{2 E_{s}}{T}} \cos \left(2 \pi f_{1} t+\psi(t, \mathbf{U})+\varphi_{0}\right), \quad t \geq 0
$$

where $E_{s}$ is the symbol energy, $T$ is the symbol period, $\varphi_{0}$ is the intial phase offset, and $f_{1}$ the asymmetric carrier frequency, which is related to the symmetric carrier frequency $f_{c}$ by $f_{1}=$ $f_{c}-(M-1) h / 2 T$. $\psi(t, \mathbf{U})$ is called the tilted (informationcarrying) phase, and is given by

$$
\psi(t, \mathbf{U})=4 \pi h \sum_{n=0}^{\rightleftharpoons} U_{n} q(t-n T), \quad t \geq 0
$$

which is assumed to be 0 at $t=0$, and the $M$-ary data sequence $\mathbf{U}$ is given by

$$
\mathbf{U}=\left(U_{0}, U_{1}, \ldots\right), \quad U_{n} \in\{0,1, \ldots,(M-1)\} .
$$

The parameter $h$ in (2) is called the modulation index. We consider only rational modulation indexes of the form $h=\boldsymbol{F} / P$ where $\boldsymbol{F}$ and $P$ are relatively prime postive integers. The phase response, $q(t)$, for CPFSK is

$$
q(t)= \begin{cases}0, & t \geq 0 \\ t / 2 T, & 0<t \geqq T \\ \frac{1}{2}, & t>T\end{cases}
$$

Using these definitions, CPFSK can be decomposed [1] into a continuous phase encoder (CPE) and a memoryless modulator $(\mathrm{MM})$ as shown in Figure 1. 


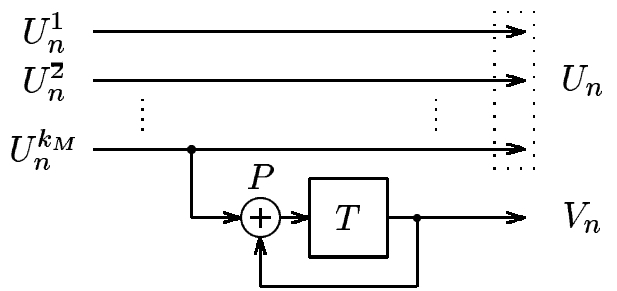

Figure 2: CPE for $P^{k_{M}}$-ary CPFSK with $h=\boldsymbol{F} / P$

The addition in the CPE is modulo- $P$, and thus the CPE is a linear encoder over the ring of integers modulo- $P\left(\mathbb{Z}_{P}\right)$, which outputs $U_{n}$ and has the state

$$
V_{n}=R_{P}\left[\sum_{i=0}^{n-1} U_{i}\right]
$$

which accounts for the accumulated phase at time $n$ due to the input data symbols from time 0 to $n-1$. $R_{P}[x]$ denotes the remainder after division by $P$ ( $x$ can be a real number or an integer). The MM generates the CPFSK signal as

$$
s(t, \boldsymbol{U})=\sqrt{\frac{2 E_{s}}{T}} \cos \left(2 \pi f_{1} t+\bar{\psi}(t, \boldsymbol{U})+\varphi_{0}\right), \quad t \geq 0
$$

where $\bar{\psi}(t, \boldsymbol{U})$, called the physical tilted phase, is defined by

$$
\begin{aligned}
& \bar{\psi}(t, \boldsymbol{U})=R_{\mathbf{2} \pi}[\psi(t, \boldsymbol{U})] \\
&\left.=R_{\mathbf{2} \pi}\left[\frac{2 \pi \boldsymbol{F}}{P} V_{n}+U_{n} \frac{t-n T}{T}\right)\right], \\
& n T \text { ? } t<(n+1) T .
\end{aligned}
$$

We can see from (5) and (7) that the number of possible tilted phase states at time $n T$ is $P$, and the physical tilted phase is equal to $2 \pi V_{n} \boldsymbol{F} / P$. The number of possible signals in the MM is $M P$.

Many coded $M$-ary CPM systems with $h=\boldsymbol{F} / P$ and $M=P^{k_{M}}$ for some integer $k_{M}$, have maximum Euclidean distance [5]. If we represent $U_{n}$ in radix- $P$ form, i.e.,

$$
U_{n}=\sum_{j=1}^{k_{M}} U_{n}^{j} P^{k_{M}-j}
$$

then the resulting $\mathrm{CPE}$ has a well defined structure, as shown in Figure 2 [1]. This form of the CPE is a rate $k_{M} / k_{M}+1$ systematic convolutional encoder over $\mathbb{Z}_{P}$, and its transfer function may be written as $\mathbf{C}(D)$ [2]. Each input $U^{j}, j=1,2, \ldots, k_{M}$, is a $P$-ary information symbol and is independently processed by a subencoder. The transfer functions $C^{j}(D)$ for the subencoders which form $\mathbf{C}(D)$ can be expressed as

$$
C^{j}(D)= \begin{cases}{[1],} & 1 \geqq j<k_{M} \\ {\left[1, \frac{D}{1-D}\right],} & j=k_{M}\end{cases}
$$

In this form, the state of the CPE is given by

$$
V_{n}=R_{P}\left[\sum_{i=0}^{n-1} U_{i}^{k_{M}}\right]
$$

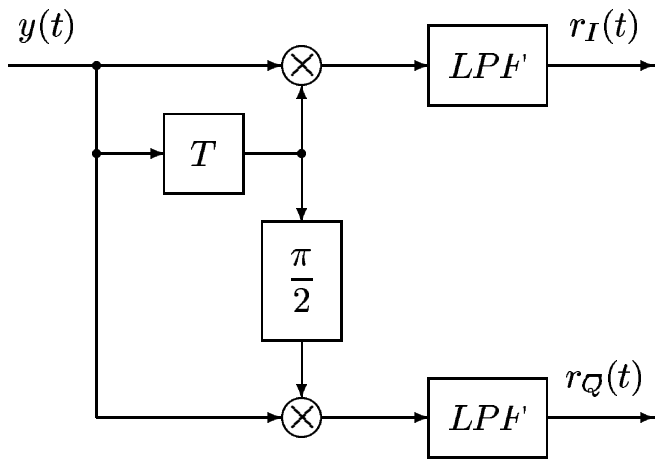

Figure 3: A receiver structure for differential demodulation

\section{Differentially Encoding CPFSK}

The accumulated symbol phase gives a CPFSK signal a "memory", which, when coherently demodulated, produces a phase trellis. A receiver can use Viterbi decoding to exploit this signal memory and improve performance.

Differential detection avoids the need for a coherent phase reference at the receiver. The idea is to use one symbol to demodulate and detect the next. The signal is differentially encoded so that the data is transmitted in the difference between symbols. If we apply this to CPFSK directly, we see that we can detect the original data, but we lose the phase trellis, and thus cannot exploit the signal memory for performance gain.

Yuan and Taylor, [4], developed a differential encoder for CPFSK that preserves the phase trellis through differential demodulation. However their encoder is designed for multi-h binary CPFSK, and does not extend to $M$-ary signals. It accepts standard binary CPM symbols, $\alpha, \alpha_{i}= \pm 1$ and outputs symbols from $\{-P, \ldots,-1,0,1, \ldots, P-1\}$, and thus is not a linear encoder.

We wish to develop an $M$-ary differential encoder for CPFSK that is also a linear encoder. This should interface well with the decomposition of CPFSK and provide greater understanding of differential CPFSK (DCPFSK). A linear encoder will also aid in the development of external error control coding for DCPFSK.

First we assume that there exists a differential encoder preceding the CPM modulator, that outputs symbols $C_{n}$. The modulated encoded signal can then be written as (using (1))

$$
\begin{gathered}
s_{e}(t)=\sqrt{\frac{2 E_{s}}{T}} \cos \left(2 \pi f_{1} t+4 \pi h C_{n} q(t-n T)+2 \pi h \sum_{i=0}^{n-1} C_{i}\right), \\
n T \text { 르 } t \text { < }(n+1) T .
\end{gathered}
$$

and we assume that $C_{i}=0, i<0$. The differential demodulator of Figure 3 receiving (11) produces the output signal

$$
\begin{aligned}
y_{d}(t) & =y_{I}(t)+j y_{Q}(t) \\
& =\frac{E_{s}}{T} \exp \left(j\left[4 \pi h\left(C_{n}-C_{n-1}\right) q(t-n T)+2 \pi h C_{n-1}\right]\right), \\
& n T \geq t<(n+1) T .
\end{aligned}
$$

A coherent demodulator receiving (1) produces

$$
y_{c}(t)=A \exp \left(j\left[4 \pi h U_{n} q(t-n T)+2 \pi h \sum_{i=0}^{n-1} U_{i}\right]\right),
$$


where $A$ is a constant. In order for the demodulated signals (12) and (13) to be equivalent, we must have

$$
C_{n}-C_{n-1}=U_{n}
$$

and

$$
C_{n-1}=\sum_{i=0}^{n-1} U_{i}
$$

Noting that $U_{n}$ only takes on $M$ different values, we can write (14) as

$$
\begin{aligned}
R_{M}\left[C_{n}-C_{n-1}\right] & =R_{M}\left[U_{n}\right] \\
R_{M}\left[C_{n}\right] & =R_{M}\left[U_{n}+C_{n-1}\right] \\
& =R_{M}\left[\sum_{i=0}^{n} U_{i}\right]
\end{aligned}
$$

where we have assumed $C_{0}=U_{0}$ with no loss of generality. Now noting that the complex exponential function operates modulo$2 \pi$ on its phase, and that $h=\boldsymbol{F} / P$, we can use $R_{a}[x+y]=$ $R_{a}\left[R_{a}[x]+y\right]$ and $R_{a b}[b x]=R_{a}[x]$ to reduce (15) to

$$
R_{P}\left[C_{n-1}\right]=R_{P}\left[\sum_{i=0}^{n-1} U_{i}\right] .
$$

Comparing (16) and (17) we can see that they differ only in the modulo base. Obviously (16) and (17) are equal if $M=P$. Now if $P<M$, then we choose $M$ as the base as we must have at least $M$ different outputs to detect the state transitions in the trellis, however $P$ must be a factor of $M$ to ensure that

$$
R_{P}\left[R_{M}\left[\sum_{i=0}^{n-1} U_{i}\right]\right]=R_{P}\left[\sum_{i=0}^{n-1} U_{i}\right] .
$$

This is not a big constraint, as it will be the case in most systems, particularly if $M=P^{k_{M}}$. However, as the DE must work over $\mathbb{Z}_{M}$ to ensure that the symbols may be recovered, the DCPE cannot be expressed as $k_{M}$ subencoders over $\mathbb{Z}_{P}$. If $P>M$ then we choose $P$ as the base in order to ensure that the trellis will have $P$ states. Thus the definition of the differential encoder (DE) is in general

$$
C_{n}=R_{B}\left[\sum_{i=0}^{n} U_{i}\right]
$$

where $B=: \operatorname{ax}\{P, M\}$. The DE is shown in Figure 4, where the addition is modulo- $B$. The DE is obviously a linear encoder over $\mathbb{Z}_{B}$. For the case when $B=P$, the $\mathrm{DE}$ can be cascaded with the CPE as shown in Figure 5.

The pair form another linear encoder over $\mathbb{Z}_{P}$ which we will call the differential continuous phase encoder (DCPE). This encoder accepts $U_{n}$ and outputs

$$
\begin{aligned}
& C_{n}=R_{P}\left[\sum_{i=0}^{n} U_{i}\right] \\
& V_{n}=R_{P}\left[\sum_{i=0}^{n-1} C_{i}\right]=R_{P}\left[\sum_{i=0}^{n-1} \sum_{k=0}^{i} U_{k}\right]
\end{aligned}
$$

to the MM.

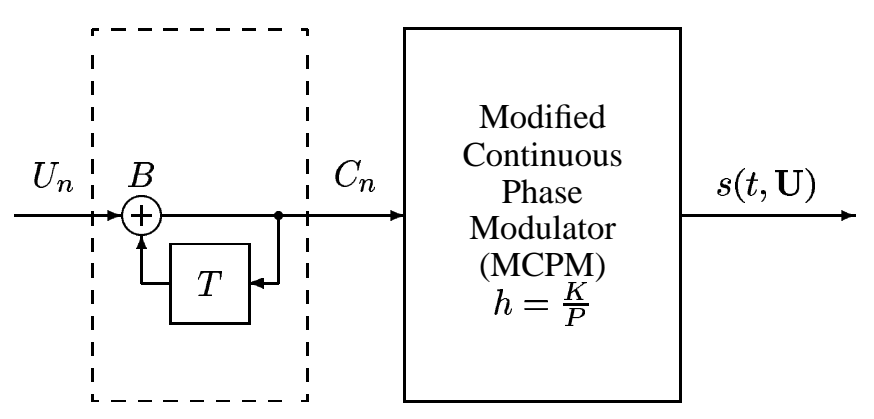

Differential Encoder (DE)

Figure 4: A differential encoder for CPFSK

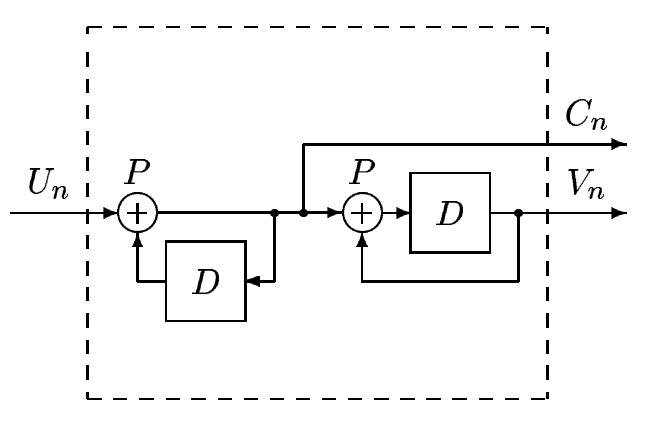

Differential CPE (DCPE)

Figure 5: The differential CPE $(P \geq M)$

\section{Discussion of DCPFSK}

Having developed a differential encoder for CPFSK, we now look in more detail at various aspects of the resulting system.

Previous work [2] [3] has shown that coding CPFSK using codes over $\mathbb{Z}_{P}$, where $P$ is the denominator of the modulation index $h$, produces better results than coded schemes using binary codes. Moreover coding over $\mathbb{Z}_{P}$ is a more natural way to code CPFSK [6]. The DCPE (for the case when $P \geq M$ ) presented in Section 3 will combine with an external linear encoder over $\mathbb{Z}_{P}$ to form an overall linear encoder. This should allow the best possible codes to be found for DCPFSK.

\subsection{Tilted Phase Trellis}

The trellis of the differentially demodulated tilted phase for DCPFSK with $M=4$ and $h=1 / 4$ is shown in Figure 6(b) and compared with that of coherently demodulated CPFSK with $M=4$ and $h=1 / 4$ shown in Figure 6(a) (the dotted lines show where the phase has wrapped modulo- $2 \pi$ ). The coherent trellis wraps around and can, therefore, be thought of as moving around a cylinder, whereas the differential trellis does not wrap, and consequently moves on a plane. Both trellises are fully connected, and we can see that the transmitted symbol can be detected from either the path or the modulo- $M$ difference between states (thus there is a one-to-one correspondence between paths and state transitions).

We now consider the case when $M=P^{k_{M}}$. The tilted phase trellises for (D)CPFSK with $M=4$ and $h=1 / 2$ are shown in Figure 7 . In both cases the part of each trellis above $2 \pi$ has been "overlaid" on the 0 state. In the coherent case (Figure 7(a)) there are still only $M$ distinct paths from each state, so we can detect the transmitted symbol from the path taken between states, but 


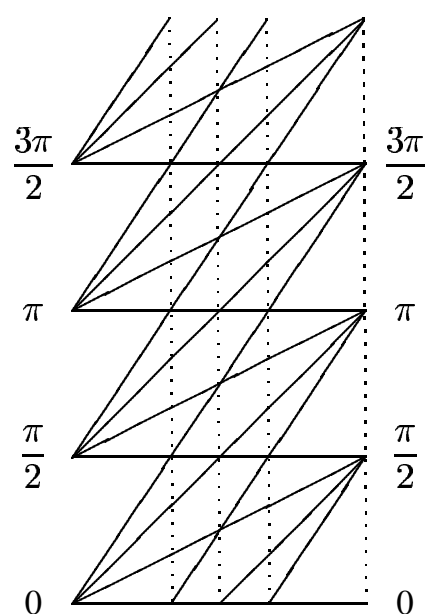

(a) coherent

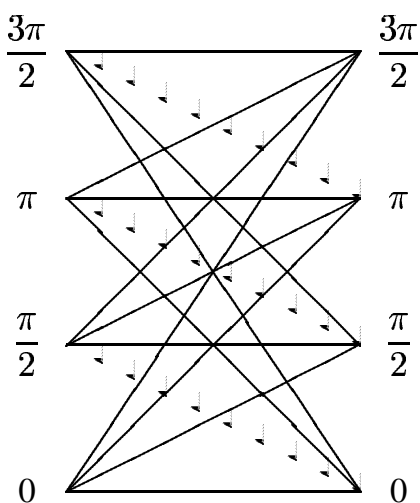

(b) differential
Figure 6: The tilted phase trellis of demodulated (D)CPFSK with $M=4$ and $h=1 / 4$.

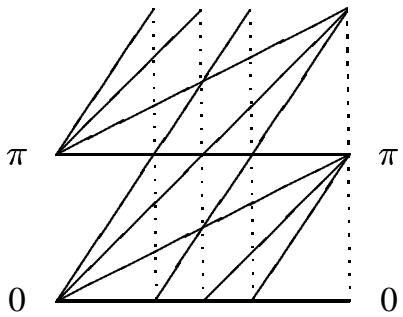

(a) coherent

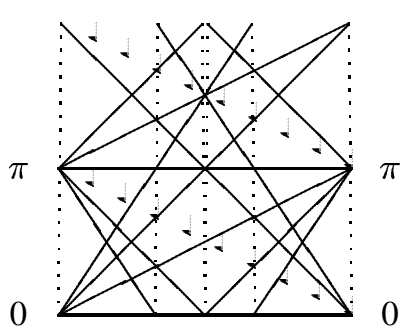

(b) differential
Figure 7: The tilted phase trellis of demodulated (D)CPFSK with $M=4$ and $h=1 / 2$.

not the difference between states as in the previous case. This is due to the fact that the set of paths paths leaving one state, differ only by a constant offset from another set of paths leaving another state, which is a consequence of the signal moving around a cylinder. In the differential trellis (Figure 7(b)), the set of paths leaving any state are different from any set of paths leaving a different state, due to the signal being forced onto a plane. Thus there are more than $M$ distinct paths between states, but each path represents a unique symbol, so we can still detect the transmitted symbol and use the trellis for Viterbi decoding.

\subsection{Squared Euclidean Distance}

The incremental squared Euclidean distance (ISED) of DCPFSK in the $n$th interval is [4]

$$
D_{n}^{2}\left(\mathbf{U}, \mathbf{U}^{\prime}\right)=\left\{\begin{array}{l}
2 E_{s}\left[1-\frac{\operatorname{sic}\left(2 \pi h \epsilon_{n}\right)-\operatorname{si\Perp }\left(2 \pi h \epsilon_{n-1}\right)}{2 \pi h\left(\epsilon_{n}-\epsilon_{n-1}\right)}\right] \\
2 E_{s}\left[1-\cos \left(2 \pi h \epsilon_{n-1}\right)\right], \quad \epsilon_{n} \neq \epsilon_{n-1} \\
\epsilon_{n}=\epsilon_{n-1}
\end{array}\right.
$$

where

$$
\epsilon_{n}=C_{n}-C_{n}^{\prime}=R_{B}\left[\sum_{k=0}^{n} U_{k}\right]-R_{B}\left[\sum_{k=0}^{n} U_{k}^{\prime}\right] .
$$

Following a derivation similar to that of [7], the minimum squared Euclidean distance (MSED) for $M$-ary DCPFSK modulation with modulation index $h=\boldsymbol{E} / P \geq 1 / 2$ where $\boldsymbol{F}$ and $P$ are relatively prime positive integers and $M=m P, m$ integer, if $M>P$, can be shown to be

$$
D_{\min }^{2}= \begin{cases}\text {. iut }\left[2 E_{s}, 4 E_{s}\left[1-\frac{\operatorname{sic} 2 \pi h}{2 \pi h}\right]\right], & M>P \\ 4 E_{s}\left[1-\frac{\operatorname{si\mu } 2 \pi h}{2 \pi h}\right], & \text { otherwise }\end{cases}
$$

which is the same result as that for coherent CPFSK [7]. This is intuitively satisfying as the DE is a scrambler [8], a one-to-one permutation of the input symbols which should not change the MSED of the system. This also means that coherently demodulated differentially encoded CPFSK will have twice the BER of CPFSK due to an single error becoming two in decoding.

Another consequence of the DE being a scrambler is that the spectrum of DCPFSK is exactly the same as that of CPFSK, which has an analytical solution (see [9]), and thus can be easily calculated. This makes the design of DCPFSK systems simpler and techniques such as those used in [5] can be used to trade off bandwidth and energy efficiency.

\section{Simulation Results}

In order to assess the performance of DCPFSK, systems with various parameters were simulated. The transmitter was based on the decomposed model of DCPFSK, with a DCPE and an MM. The channel used was the AWGN channel. The receiver consisted of a noise limiting filter in front of the differential demodulator of Figure 3 followed by a $P$-state Viterbi processor with a decision depth large enough to ensure that errors due to truncation were negligible. The Viterbi processor used Euclidean distance between the received signal and possible paths in the differential phase trellis to form a metric. Although this is not the optimal metric in the presence of differential noise, it is a simple metric and approaches the optimal metric at high SNR. It was found that the bandwidth of the noise-limiting filter at the front end of the receiver must be chosen carefully to ensure the best possible performance. For $M=2$ this was found to be 0.75 times the symbol rate $r_{s}$, and for $M \in\{4,8,16\}$ it was found to be in the range $2-3$ times $r_{s}$.

The bit error rates (BERs) for various DCPFSK schemes obtained in the simulations are shown in Figure 8. There is an obvious degradation of BER as $M$ increases, this is due to the decreasing modulation index, or equivalently, the decreasing MSED. However, the spectrum of a DCPFSK signal with $h=1 / M$, is comparable to that of MSK. So as we increase $M$, we increase the data rate without bandwidth expansion. These losses are very similar to those found in PSK as $M$ is increased. The $E_{b} / N_{0}$ required to obtain a BER of $10^{-3}$ for both CPFSK and DCPFSK is shown in Table 1. This highlights the definite performance loss in going from CPFSK to DCPFSK which increases with $M$, however for $M \geq 4$ it seems to be fairly constant, which again agrees with PSK results. 


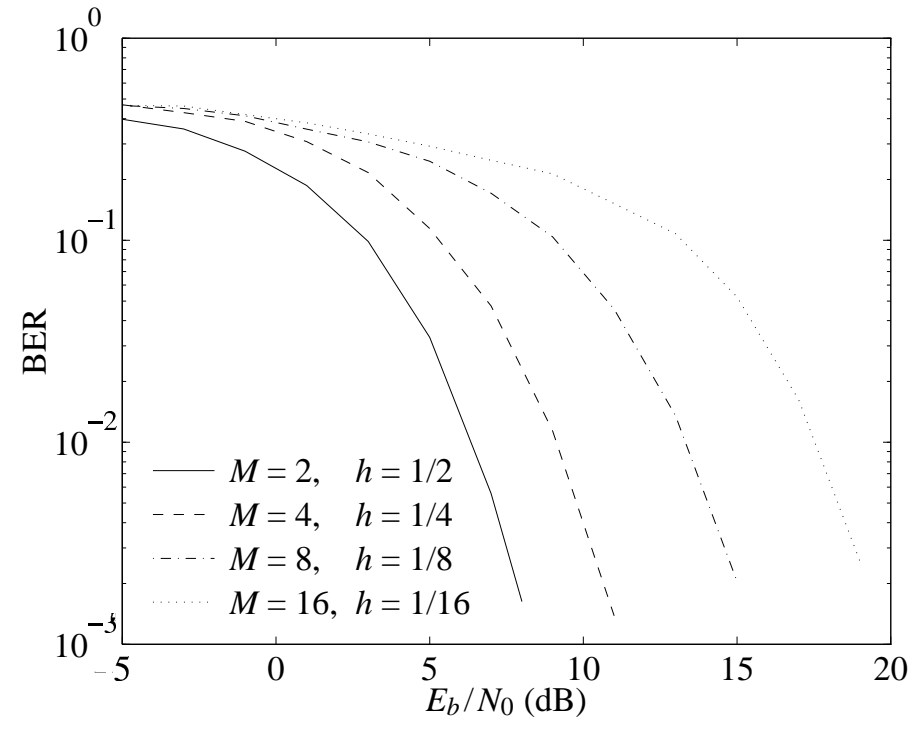

Figure 8: Simulated BER vs $E_{b} / N_{0}$ performance for DCPFSK with $h=1 / M$

\begin{tabular}{|c|c|c|}
\hline & \multicolumn{2}{|c|}{$E_{b} / N_{0}$ in dB } \\
\hline$M$ & CPFSK & DCPFSK \\
\hline 2 & 7.2 & 8.3 \\
4 & 8.1 & 11.1 \\
8 & 12.1 & 15.6 \\
16 & 16.7 & 19.9 \\
\hline
\end{tabular}

Table 1: $E_{b} / N_{0}$ required to acheive a BER of $10^{-3}$

\section{Conclusions}

We have developed a differential encoder for $M$-ary differential CPFSK which preserves the phase trellis through differential demodulation. For schemes with $P \geq M$, the DE combines with the CPE to form another linear encoder over $\mathbb{Z}_{P}$. We call this the DCPE and it and the MM form the decomposed model of DCPFSK. The DCPE will allow coding on $\mathbb{Z}_{P}$ to be developed specifically for DCPFSK. Note that $P \geq M$ comprises the majority of interesting schemes as increasing $M$ above $P$ incurs a bandwidth penalty. We also note that there is room for improving the performance of DCPFSK by using an optimal receiver structure that is specifically designed for DCPFSK.

\section{References}

[1] B. Rimoldi, "A decomposition approach to CPM", IEEE Transactions on Information Theory, vol. 34, no. 2, pp. 260270, Mar. 1988.

[2] R. H. Yang and D. P. Taylor, "Trellis coded continuous phase frequency shift keying with ring convolution codes", IEEE
Transactions on Information Theory, vol. 40, no. 4, pp. 10571067, July 94.

[3] B. Rimoldi and Q. Li, "Coded continuous phase modulation using ring convolutional codes", IEEE Transactions on Communications, vol. 43, no. 11, pp. 2714-2720, Nov. 1995.

[4] L. Yuan and D. P. Taylor, "Differential detection of differentially encoded multi-h CPM using viterbi decoding", Tech. Rep. 250, Communication Research Laboratory, McMaster University, Canada, May 1992.

[5] B. Rimoldi, "Design of coded CPFSK modulation systems for bandwidth and energy efficiency", IEEE Transactions on Communications, vol. 37, no. 9, pp. 897-905, Sept. 1989.

[6] J. L. Massey and T. Mittleholzer, "Convolutional code over rings", in Proceeding of Fourth Joint Swedish-USSR Int. Workshop on Information Theory, Aug. 1989, pp. 14-18.

[7] B. Rimoldi, "Exact formula for the minimum squared euclidean distance of CPFSK", IEEE Transactions on Communications, vol. 39, no. 9, pp. 1280-1282, Sept. 1991.

[8] G. D. Forney Jr, "Convolutional codes I: Algebraic structure", IEEE Transactions on Communications, vol. IT-16, no. 6, pp. 720-738, Nov. 1970.

[9] J. B. Anderson, T. Aulin, and C-E. Sundberg, Digital Phase Modulation, Plenum, 1986. 